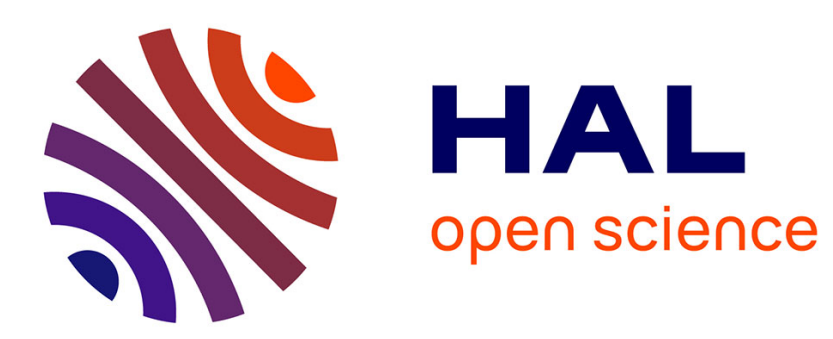

\title{
Transient Phenomena in Plasma Torches and in Plasma Sprayed Coating Generation
}

\author{
Pierre Fauchais, J. Coudert, Michel Vardelle
}

\section{To cite this version:}

Pierre Fauchais, J. Coudert, Michel Vardelle. Transient Phenomena in Plasma Torches and in Plasma Sprayed Coating Generation. Journal de Physique IV Proceedings, 1997, 07 (C4), pp.C4-187-C4-198. 10.1051/jp4:1997414 . jpa-00255570

\section{HAL Id: jpa-00255570 https://hal.science/jpa-00255570}

Submitted on 1 Jan 1997

HAL is a multi-disciplinary open access archive for the deposit and dissemination of scientific research documents, whether they are published or not. The documents may come from teaching and research institutions in France or abroad, or from public or private research centers.
L'archive ouverte pluridisciplinaire HAL, est destinée au dépôt et à la diffusion de documents scientifiques de niveau recherche, publiés ou non, émanant des établissements d'enseignement et de recherche français ou étrangers, des laboratoires publics ou privés. 


\title{
Transient Phenomena in Plasma Torches and in Plasma Sprayed Coating Generation
}

\author{
P. Fauchais, J.F. Coudert and M. Vardelle
}

Université de Limoges, Faculté des Sciences, Laboratoire de Matériaux Céramiques et Traitements de Surface, UPRES A 6015, 123 avenue Albert Thomas, 87060 Limoges cedex, France

\begin{abstract}
D.C. plasma torches are more and more widely used for different applications among which cutting, welding and spraying are probably the most developed. For a long period it is mainly their static characteristics which have been considered and their applications were studied on a macroscopic scale.

However many transient phenomena occur, the systematic study of which has started only a few years ago. To limit the discussion we present only the case of plasma spraying with :

- the transient phenomena, at the anode, and their evolution with the torch working parameters and the electrodes erosion,

- the consequences of these fluctuations on plasma jet temperature and velocity fields as well as mean electric field in the arc column,

- the characterization of the molten particles flattening and resulting splat cooling and consequences on coating properties.
\end{abstract}

\section{INTRODUCTION}

Plasma spraying is now a versatile technology that has been successful as a reliable cost-effective solution for many industrial problems. It allows the spraying of a wide range of high performance materials, from superalloys and refractory intermetallic compounds to ceramics with continuously increasing commercial applications[1]. In most cases (99\%) plasma spraying is achieved by using d.c. plasma torches which will be described summarily in section 2. The solid particles, with diameters ranging between 20 and $60 \mu \mathrm{m}$, are injected orthogonally to the plasma jet, either upstream or downstream of the nozzle exit with the help of a carrier gas which is mainly argon[2-3]. Their flow rate is between 2 and $6 \mathrm{~kg} / \mathrm{h}$. The coating is built by layering of splats resulting from the flattening of particles in a molten state upon a substrate disposed at a distance, which for atmospheric plasma spraying (APS), is between 80 and $120 \mathrm{~mm}$. The flattening time is in the 1 to $1.8 \mu \mathrm{s}$ range and, on a smooth surface (mean roughness $\mathrm{Ra}<0.1 \mu \mathrm{m}$ ), the resulting splat which diameter, assuming a disk shape, is 3 to 6 times that of the impacting particle solidifies in times between a few $\mu \mathrm{s}$ to $20 \mu \mathrm{s}[4,5]$. Unmolten particles rebound from the substrate or coating and finally the deposition efficiency is in the range 40 to $70 \%$. Especially in industrial conditions, process parameters to produce sound coatings are specified in terms of stationary controllable parameters associated with the spray equipment, e.g. arc current, plasma gas composition and flow rate, stand-off distance, particle carrier gas flow rate, particle injector position, particle mass flow rate... They are typically established based on experiment or experience.

If they were known[6] 25 years ago, transient phenomena taking place in plasma torches were rediscovered by scientists about 5 years ago but are still ignored by industry. This rediscovery is due to the recent availability of investigation means such as fast numerical oscilloscopes and powerful software for signal treatment.

In the eighties McPherson[7] has underlined that contact between the layered splats was far from being perfect and conditioned the resulting coating thermomechanical properties[7-8]. The only way to understand which parameters control the contact between the layered splats is, for an impacting particle with a given velocity, diameter and temperature, to follow the evolution of its temperature and diameter during flattening and of the corresponding splat temperature upon cooling. Such studies require fast micro-pyrometers (50-100 ns response time) that became available only a few years ago[9].

This presentation will be devoted to an overview of our actual knowledge of :

- the transient phenomena especially at the anode and their consequences on plasma jet temperature and velocity fields, as well as arc column mean electric field, 
- the measurement of molten particles flattening and the resulting splat cooling. Finally it will be shown how these microscopic results correlate with coating macroscopic properties.

\section{TRANSIENT PHENOMENA IN D.C. PLASMA TORCHES}

\subsection{Used plasma torches}

The most common configuration is a stick type thoriated $(2 \mathrm{wt} \%)$ tungsten cathode associated with a nozzle shaped anode serving also as a constrictor. Fig. 1 shows a typical configuration. In most cases the nozzle has a conical shape followed by a cylindrical duct.

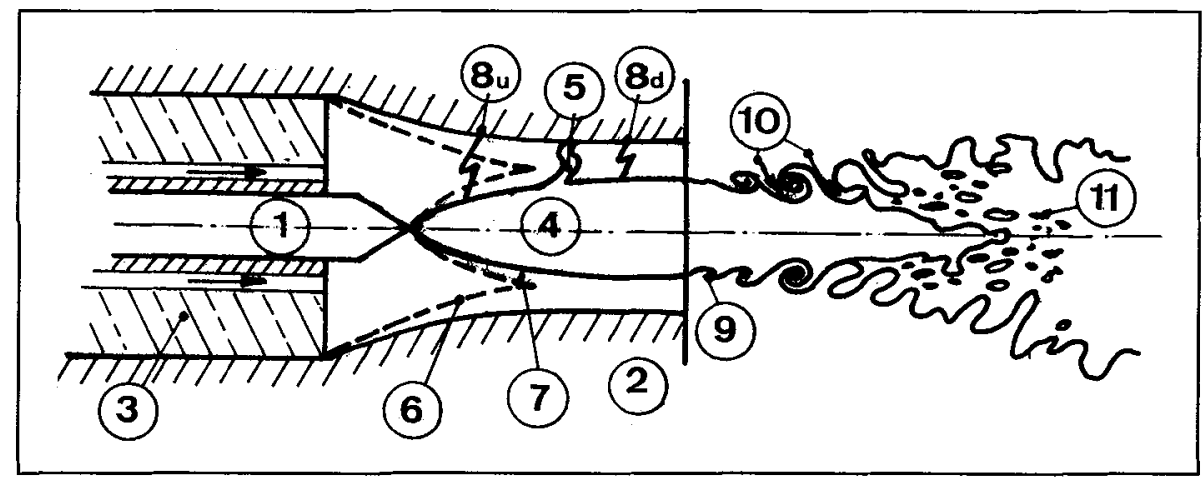

Figure 1 : Plasma spray torch operation at atmospheric pressure showing (1) stick type cathode with a conical tip, (2) anode, (3) insulating material with axial injection, (4) plasma column, (5) arc column attachment to the anode wall, (6) cold boundary layer, (7) hot boundary layer, (8) short circuits upstream (u) and downstream (d) of the arc root, (9) vortex ring coalescence, (10) cold gas engulfment, (11) entrained cold gas bubbles. The injected plasma forming gas is indicated with the arrows along the cathode.

The plasma forming gas is injected either axially (as shown in fig. 1) or as a vortex around the cathode or along its conical tip... The used plasma gases are $\mathrm{Ar}-\mathrm{H}_{2}$ (5 to $25 \mathrm{vol} \%$ ), $\mathrm{N}_{2}-\mathrm{H}_{2}$ (5 to 20 vol\%) and $\mathrm{Ar}-\mathrm{He}(40$ to $80 \mathrm{vol} \%)$. The first gas ( $\mathrm{Ar}$ or $\mathrm{N}_{2}$ ) gives the jet momentum and the second $\left(\mathrm{H}_{2}\right.$ or $\left.\mathrm{He}\right)$ improves its thermal conductivity.

The plasma column develops between the cathode and the anode where an electrically conducting gas at $\mathrm{T}>8000 \mathrm{~K}$ and $10^{5} \mathrm{~Pa}$ (for the considered plasma gases) can be found. The radius of the plasma column, also termed the electrical radius $r_{e}$, depends on the plasma gas flow rate and composition, cooling conditions at its fringes and arc current.

The arc root strikes at the nozzle wall when the cold boundary layer close to the anode wall is sufficiently heated to reduce the arc column constriction. This configuration is called "the free arc length". The voltage depends on the arc length linked to arc current, nozzle diameter, gas composition and flow rate.

Typical operation conditions are : anode-nozzle internal diameter (i.d.) D between 5 and $8 \mathrm{~mm}$, total gas flow rates between 35 and 120 standard liters per minute (slm) and power levels between 20 and $60 \mathrm{~kW}$ with arc currents generally below $700 \mathrm{~A}$.

\subsection{Transient phenomena at the anode}

In this plasma torch configuration, if the arc geometry is rather simple in the vicinity of the cathode tip, where the arc column shows a cylindrical symmetry, the arc exhibits a complex and changing shape in the arc-anode attachment region, under the influence of gas dynamic and magnetic body forces.

The modifications of the morphology of the arc are associated with typical features of the recorded voltage which gives a sawtooth shaped waveform with quasi-linear increasing ramps followed by negative voltage jumps (fig. 2). In all the experiments performed the arc current varies very little when the voltage jumps to lower values : small peaks show up which amplitude is less than $2.5 \%$ of the regulated mean arc current. The slope of the ramps indicates the rate at which the arc and the imbedded current lines 
are stretched and lengthened by the flow. Each of the lengthening phases is limited by an electrical breakdown through the cold and electrically insulating gas layer surrounding the arc. Each breakdown initiates a short circuit, together with a new arc attachment at the nozzle wall, and is associated with a negative jump of the voltage. This situation corresponds to the so called "restrike mode"[6] and has been reported in the past by different experimental investigators[10-12].

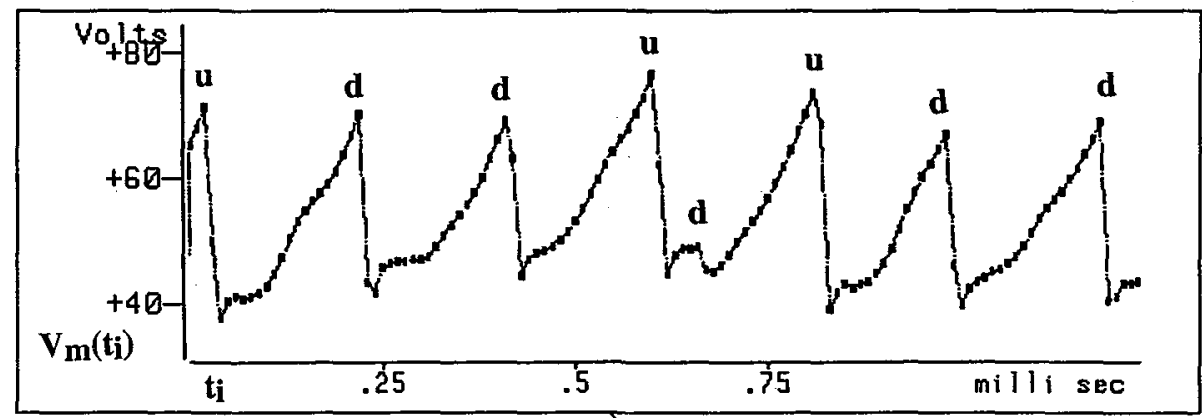

a)

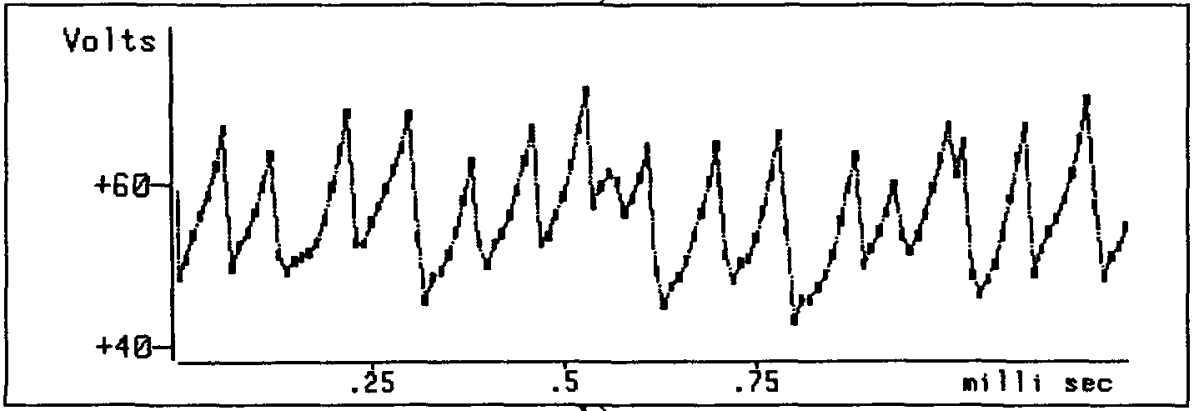

b)

Figure 2 : Typical voltage signals obtained with a $8 \mathrm{~mm}$ i.d. nozzle with $\mathrm{Ar}-\mathrm{H}_{2}(25$ vol\%) 60 slm for two different arc currents : a) $209 \mathrm{~A}$; b) $604 \mathrm{~A}$.

Such transient phenomena can be studied with the help of a Fast Fourier Transform (FFT) algorithm[13,14] or a statistical study of the tendencies shown by the characteristics of the arc voltage fluctuating components $[15,16,17]$. With FFT, characteristic frequencies $f$ can be isolated and their variations with torch working parameters : arc current $\mathrm{I}(\mathrm{A})$, gas flow rate $\mathrm{m}_{\mathrm{g}}^{\circ}(\mathrm{kg} / \mathrm{s})$, gas composition, anode-nozzle internal diameter $\mathrm{D}(\mathrm{mm})$, plasma forming gas injection mode can be studied. With the statistical study of the voltage signals (see fig. 2) the minimum voltages $V_{m}$ (local minimum value of the arc voltage immediately after breakdown) the slopes of the voltage ramps, the life times of the arc root $\tau_{i}\left(\tau_{i}=t_{i+1}-t_{i}\right)$, the voltage jumps $\Delta \mathrm{V}$, the "upstream" (u) breakdowns $\left(\mathrm{V}_{\mathrm{m}}\left(\mathrm{t}_{\mathrm{i}}\right)<\mathrm{V}_{\mathrm{m}}\left(\mathrm{t}_{\mathrm{i}-1}\right)\right)$ and the "downstream" (d) breakdowns $\left(V_{m}\left(t_{i}\right)>V_{m}\left(t_{i-1}\right)\right.$ (see fig. 2.a) can also be linked to the arc working parameters. The obtained results[13-18] for $\mathrm{Ar}-\mathrm{H}_{2}, \mathrm{Ar}-\mathrm{N}_{2}, \mathrm{~N}_{2}$ and $\mathrm{Ar}-\mathrm{He}$ plasmas show that the restrike mode is mainly governed by the thickness and stability of the "cold" gas layer surrounding the plasma column. The thicker will be this layer the higher will be the voltage jumps $\Delta \mathrm{V}$, the lower the characteristic frequency and the arc root lifetime $\bar{\tau}$.

This is illustrated in fig. 3 showing the evolutions of $\Delta V$ and $\tau$ with the arc current $I$ (the electrical radius $r_{e}$ increases with $\left.I[19]\right)$. 


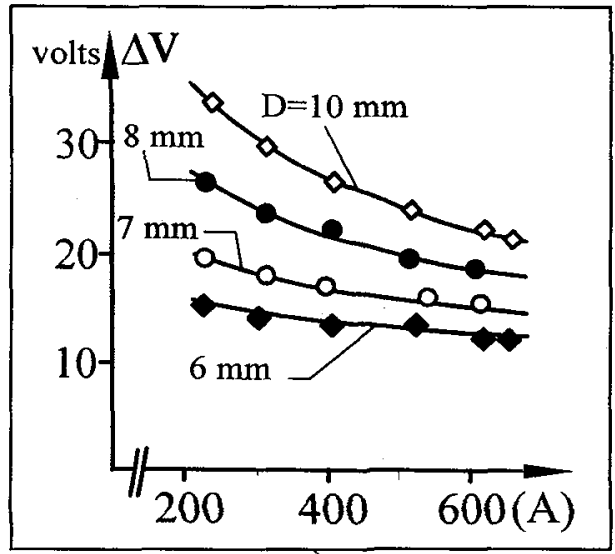

a)

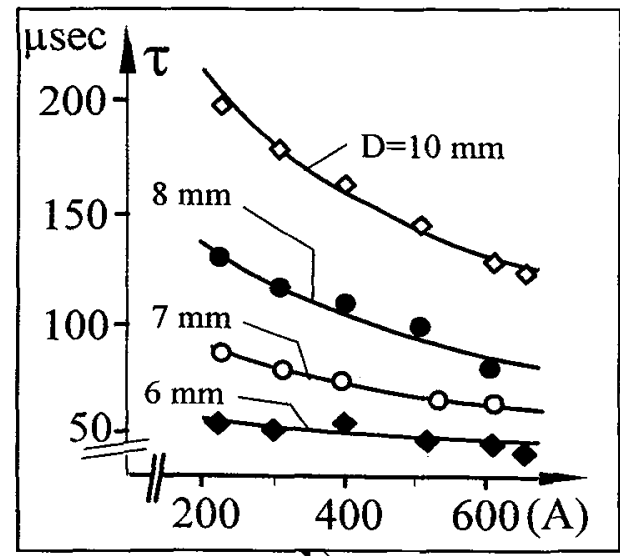

b)

Figure 3 : Evolution of a) voltage jump $\Delta V$ and b) arc root life times with arc current for four anode nozzle i.ds. for an Ar- $\mathrm{H}_{2}$ plasma : $45 \operatorname{sim} \mathrm{Ar}, 15 \sin \mathrm{H}_{2}$ gas injection along the cathode tip.

In fact when representing the arc root lifetime with the corresponding voltage minima two distributions are obtained as shown in fig. 4 corresponding respectively to the upstream and downstream restrikings (see fig. 2.a). The highest lifetimes are obtained for the high voltage minima which means that the erosion is enhanced in these regions as confirmed by the wear of the anode material.

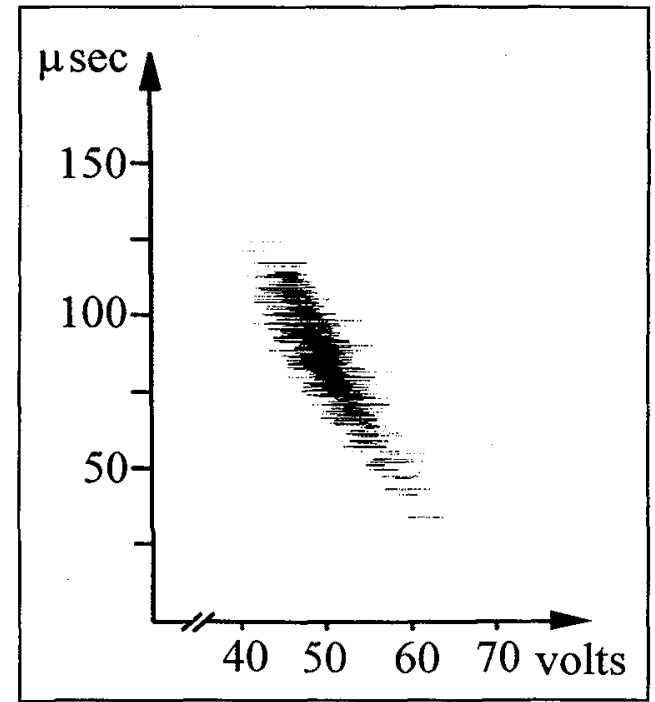

a)

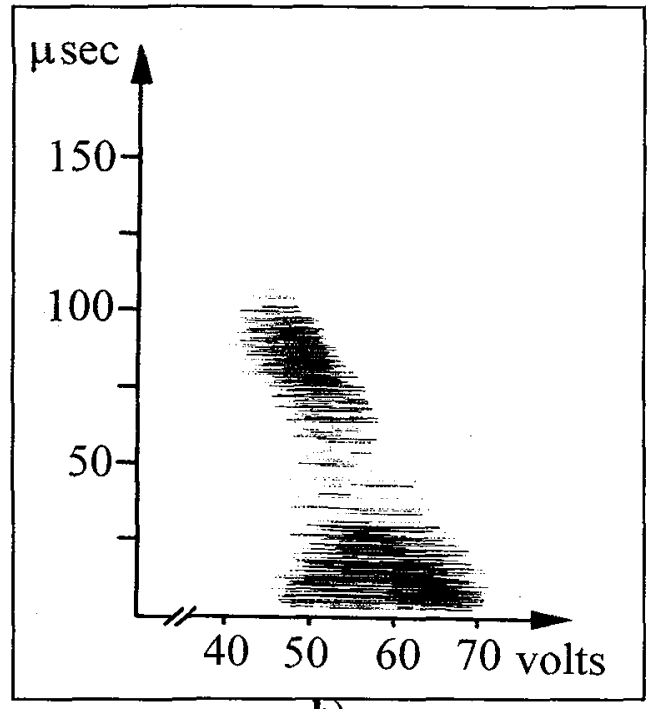

b)

Figure 4 : Individual arc root lifetimes evolution with their corresponding voltage minima : a) downstream jumps ; b) upstream jumps. $d=7 \mathrm{~mm}, 45 \operatorname{sim} \mathrm{Ar}, 15 \operatorname{sim} \mathrm{H}_{2}, \mathrm{I}=600 \mathrm{~A}$.

Semi-empirical correlations, obtained through dimensionless analysis[20,18], allow to relate the working parameters $\left(\mathrm{I}, \mathrm{d}, \mathrm{m}^{\circ} \mathrm{g}\right.$ ) to arc root lifetimes, ramp slopes and voltage jumps for a given gas mixture. They are of the type :

$$
\bar{\tau}_{\mathrm{u}}=\mathrm{K}_{\mathrm{u}} \times \mathrm{m}^{\circ} \mathrm{g}^{-0.32} \times \mathrm{I}^{-0.69} \text {. D2.0 }
$$

where $\mathrm{K}_{\mathrm{u}}=14.6$ for $\mathrm{Ar}-\mathrm{H}_{2}(25 \mathrm{vol} \%)$ and 12.7 for $\mathrm{N}_{2}$.

A similar relationship is obtained for the downstream lifetimes $\bar{\tau}_{\mathrm{d}}$. With correlations better than 0.95 they show that the approach of the phenomena is good.

The values of the characteristic frequencies deduced from the FFT are somewhat lower, especially for high arc currents, than those calculated from arc root mean lifetimes. This is due probably to the fact that FFT favors the higher amplitudes of the voltage signal while the statistical analysis takes into account 
all the peaks. As it can be observed in fig. 2.b that, at 600 A the voltage jump amplitudes are more irregular than those obtained at lower arc current (see fig. 2.b).

The way the plasma forming gas is injected has a great importance on $f, \tau, \Delta V[14] . .$. When the gas is injected axially or along the cathode tip the arc column expands closer to anode-nozzle wall and the cold gas layer around the arc determines the restrike as explained in the previously described results. The higher is arc gas flow rate, the thicker the boundary layer and the lower the restrike frequency (see fig. 5.a). With vortex injection the influence of the gas flow rate is the opposite. For higher gas flow rate the frequency peak increases with increasing gas flow rate (see fig. 5.b). Two opposing effects are present in

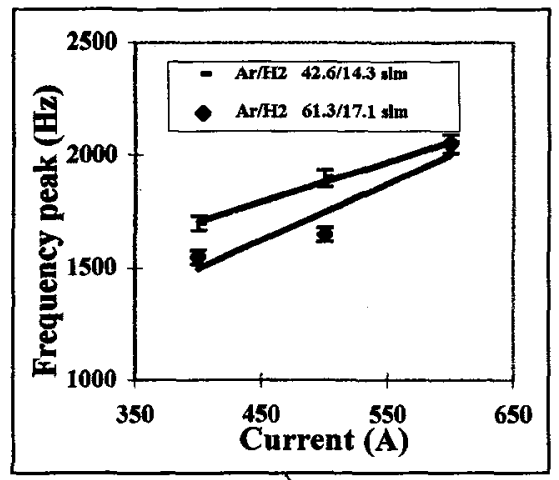

a)

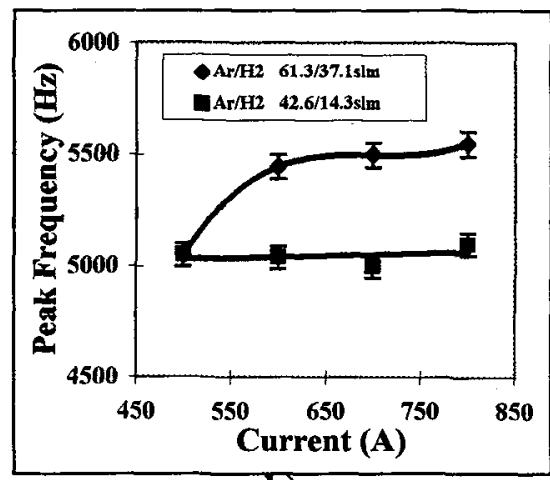

b)

Figure 5 : Evolution with arc current of the characteristic FTT frequency for two different types of plasma forming gas injectors : a) axial injection ; b) vortex injection, anode $\mathrm{D}=7.2 \mathrm{~mm}$, with two gas flow rates $42.6 \mathrm{slm} \mathrm{Ar}$ and $143 \mathrm{slm} \mathrm{H}_{2}$ and $61.3 \operatorname{sim} \mathrm{Ar}$ and $17.1 \mathrm{sim} \mathrm{H}_{2}$ respectively.

this case. First increasing $\mathrm{m}^{\circ}$ induces a constriction of the arc column which can be verified by the increase of the arc mean voltage. The consequence is reduced restrike probability and longer anode attachment. On the other hand, increasing the gas flow rate leads to higher flow velocity especially in the cold boundary layer. Because of this the connection between the arc column and the anode surface is pushed downstream faster, yielding faster and more frequent breakdowns.

The last parameter is of course the gas composition. Diatomic gases either pure $\left(\mathrm{N}_{2}\right)$ or added to Argon $\left(\mathrm{H}_{2}\right.$ or $\left.\mathrm{N}_{2}\right)$ constrict drastically the plasma column due to their high thermal conductivity[20] and with them the restrike mode is well defined. On the contrary in case of Ar-He mixtures the mean voltage is less than $40 \mathrm{~V}$, the voltage fluctuations have very small amplitudes and the restrike does not obviously appear on the time resolved signals which lead to a "noisy" power spectrum. This is due to the poor constriction of the column with these gases.

\subsection{Electrode erosion}

\section{a) Anode}

In their experiments with flat anodes with a smooth surface $(\mathrm{Ra}<0.5 \mu \mathrm{m})$ Wutzke et al[6] have shown that it was the arc root itself which was sliding on the anode surface before a new spot was created by a short circuit shortening the arc. Later on Zhukov et al[11], by using real plasma torches with a cylindrical anode equipped with observation windows, have demonstrated that the arc root once it was created by a short circuit did not move at all. It was the connecting column between the arc root and the main plasma column which was stretched downstream as long as a short circuit did not create a new arc root. It is worth to note that in both cases, moving arc root or stretching connecting column result in the same saw-tooth shape of the arc voltage. A recent experiment was performed at the laboratory[22] with the arc transferred to a flat anode orthogonal to the cathode axis, arc stabilized by gas injection along the conical tip of the cathode and working in the anode jet dominated mode in order to simulate a behavior identical to that of the plasma torch where an anode jet always exists at the anode[19]. It has been shown that when moving the cathode parallel to the anode with a new smooth ( $\mathrm{Ra}<0.1 \mu \mathrm{m}$ ) copper anode surface the arc moves as a whole. As soon as the surface is oxidized or roughened by the arc and, depending on cathode-anode relative velocity, the arc root does not move anymore and a small connecting column between the arc root and the arc main column is stretched. When the voltage drop in it becomes too high a short circuit occurs between the anode and the main plasma column and a new connecting 
column is formed in about $1 \mu$ s. The voltage signal shape is exactly the same as that shown in fig. 2 . This is probably what happens in the d.c. torch. At the beginning during the first working hour the arc root moves at the surface and the distributions $\tau=f(V \min )$ are very dispersed with relatively low values of $\tau$. The observation of the anode shows a clean surface with a well distributed slight erosion. Later on, for example after $10 \mathrm{~h}$, the distribution is well defined as that shown in fig. 4 and the electrode erosion is distributed along an area which length is about $1.5 \mathrm{x} \mathrm{D}$. The depth of the eroded zone is about $0.2-0.5$ $\mathrm{mm}$. When time increases $\tau$ increases too while the amplitude of $\Delta V$ increases and the depth of the eroded area increases drastically (a few $\mathrm{mm}$ after 20 to $50 \mathrm{~h}$ depending on working conditions).

\section{b) Cathode}

The high temperatures $(\mathrm{T}>3800 \mathrm{~K})$ observed at the thoriated $(2 \mathrm{wt} \%)$ tungsten cathode tip[23-25] promotes the dopant diffusion and evaporation[24-27]. After less than about $1 \mathrm{~h}$ the temperature of the cathode tip is that of pure tungsten (almost $1000 \mathrm{~K}$ higher than that with the dopant) and its erosion increases drastically. Of course it depends on electrode diameter and tip shape controlling the heat withdrawal and temperature gradients and on gas composition, diatomic gases enhancing the constriction of the arc root and thus the erosion. The erosion that occurs is a neck erosion below the tip which falls off after a while[26]. Thus, starting with a sharp tip, after a few hours working the cathode shape is that of a truncated cone, the extremity diameter of which increases with time.

\subsection{Consequences of fluctuations and erosion}

Each time a new arc root is created, a plasma bubble, the volume in which the electrical conductivity is high enough, begins to grow (see fig. 6[15,281). This plasma bubble is then pushed downstream by the plasma flow partly due to the gas feeding and partly by thermal expansion and M.H.D. forces. During the growth of the plasma bubble, the current lines are lengthened (voltage ramps in fig. 2). When a new arc root is created, the arc is shunted and voltage drops and the bubble partly disconnected from the plasma giving rise to a flying puff travelling with the flow. The shape of the puff, even though not clearly defined, is gradually modified by dissipative phenomena. Thus the generated plasma flow may be thought of as a rather inhomogeneous medium made up of puffs of hot gas surrounded by colder layers.

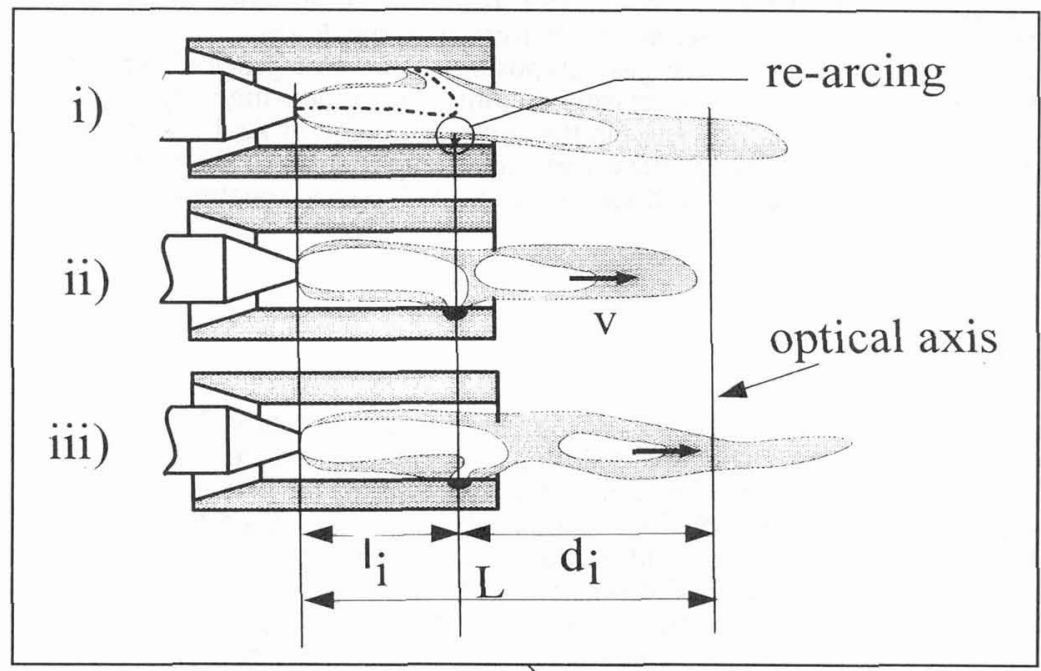




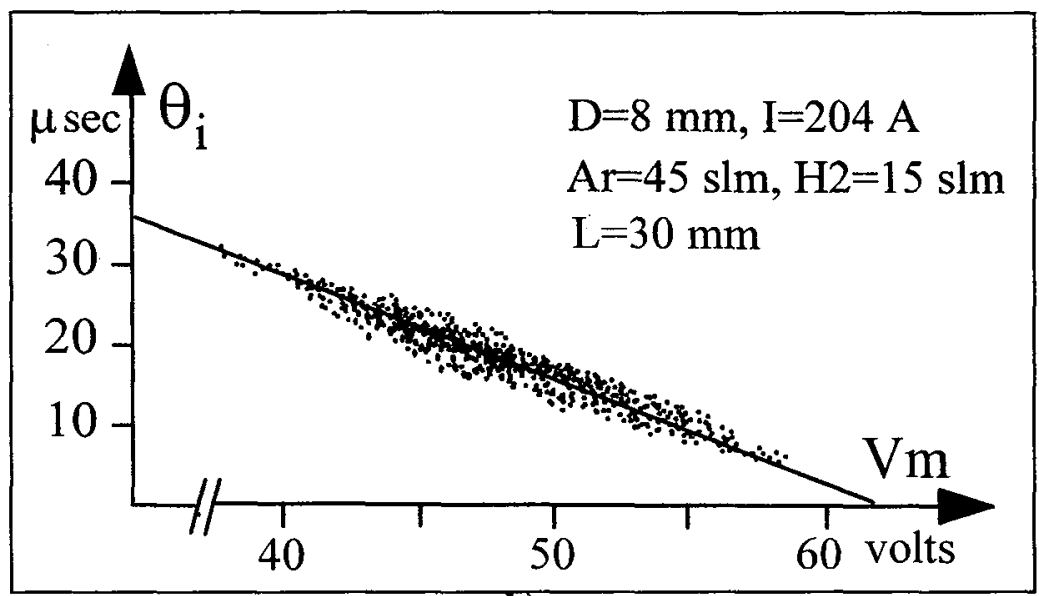

b)

Figure $6:$ a) Scheme of plasma bubble generation : i) just before rearcing, ii) just after rearcing and iii) lengthening phase ; b) Plot of time delay $\Theta$ versus the minimum voltage.

In the set-up developed by Coudert et al[28] the image of the jet was split and focused with a known magnification, in two planes where the wavelength integrated light was sampled by two optical fibers. The distance between the two fibers was known with a precision of $2 / 100 \mathrm{~mm}$. When a bright puff of plasma crossed the optical system line of sight it generated a peak clearly associated with a ramp of voltage variation. The observation of the fluctuating optical signal generated suggested that only slowly varying details were preserved and that the useful information was kept in low frequency band pass. After careful signals treatment it was shown that the low-band-pass filtered signals were cross-correlated giving rise to a function exhibiting a rather well marked and shifted maximum. A mean value of the velocity was determined as well as its fluctuating components, limited to a $500 \mathrm{~Hz}$ band pass due to the specificity of the method, fig. $7[29]$ shows two axial velocity distributions measured at the nozzle exit.

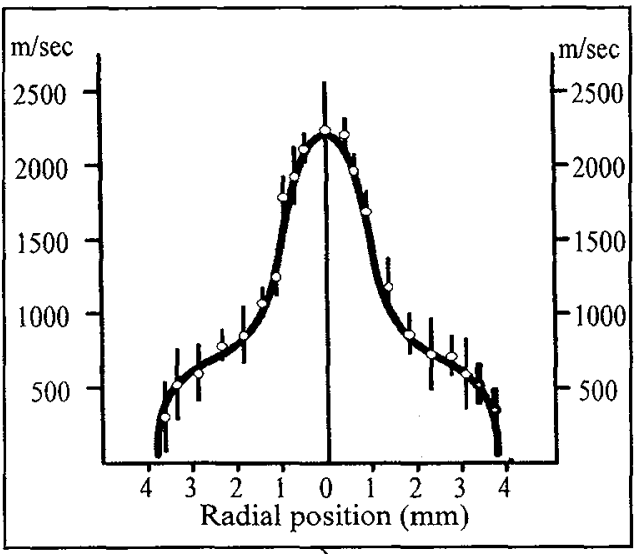

a)

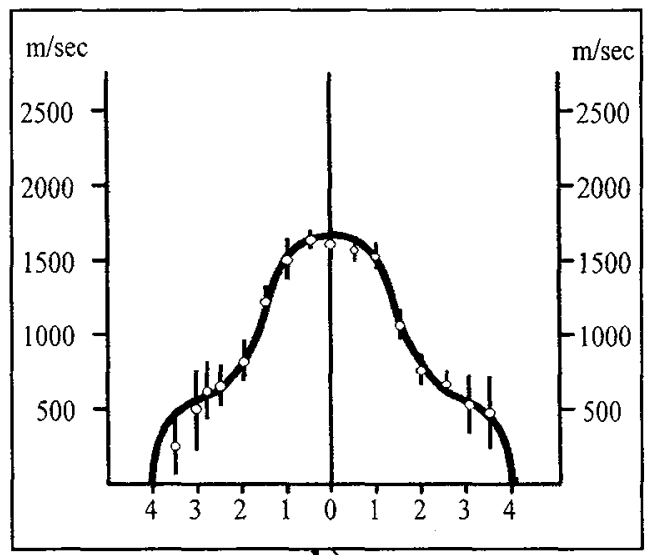

b)

Figure 7 : Radial velocity profile of a d.c. plasma torch $\left(45 \operatorname{sim} \mathrm{Ar}, 15 \operatorname{sim} \mathrm{H}_{2}, \mathrm{I}=600 \mathrm{~A}, \mathrm{D}=7 \mathrm{~mm}\right.$ ) for two working times of the torch : a) $t=1 \mathrm{~h}, \mathrm{~b}) \mathrm{t}=12 \mathrm{~h}$.

Three remarks can be made :

i) With the $\mathrm{Ar}-\mathrm{H}_{2}(25 \mathrm{vol} \%)$ mixture used at $14000 \mathrm{~K}$ axial temperature at the nozzle exit the sound velocity $\mathrm{a}=2900 \mathrm{~m} / \mathrm{s}^{[30]}$ and thus the measured velocities are subsonic.

ii) The short time scale fluctuations, represented by the height of the bars show an almost constant value in the central part of the jet and increases in its fringes due to turbulences. It is the same for measurement along the axis $\mathrm{z}$ of the torch. For $\mathrm{z} \geq 30 \mathrm{~mm}$ corresponding to the plasma core tip (in the 
working conditions of fig. 7) the fluctuations increase drastically due to instabilities generated by the engulfment of cold surrounding gas[31].

iii) The cathode erosion reduces the plasma jet maximum velocity and broadens its radial distribution. This erosion has a stabilizing effect after a few hours.

Voltage fluctuations have also an important influence on temperature distributions measured by emission spectroscopy : mean value of an exponential term does not give the mean value of the exponent! Thus the signal has also to be treated[32,17] to get stationary and fluctuating components of the temperature, the stationary temperature component being smaller than temperature value deduced from a time averaged light intensity signal. Contrarily to what happens for velocity, temperature profiles seem to be unaffected by the electrode erosion[29].

At last by comparing the voltage and light fluctuations signals it is possible to determine the mean electric field within the arc column as well as the mean position of the erosion inside the anode-nozzle[33].

The time delay between light and voltage signals is given by:

$$
\Theta=d_{i} / v
$$

where $v$ is the velocity of the plasma bubble and $d_{i}=L-\ell_{i}$ (see fig. 6 ) where $L$ is the distance between the cathode tip and the optical axis and $\ell_{i}$ is the instantaneous arc root position.

For the minimum voltage :

$$
V_{m}=\bar{E} \times \ell_{i}+V_{A}+V_{K}
$$

where $\bar{E}$ is the mean electrical field within the arc column, $V_{A}$ and $V_{K}$ the anodic and cathodic voltage drops respectively. In these conditions the measured values of $\Theta$ are related to $\overline{\mathrm{E}}$ by :

$$
\Theta=\frac{L}{v}-\frac{V_{m}-V_{A}-V_{K}}{v \cdot \bar{E}}
$$

A plot of $\Theta$ as a function of $V_{m}$ (see fig. 6.b) gives a straight line which at its intercepts with $\Theta$ axis gives $\mathrm{L} / \mathrm{v}$ thus $\mathrm{v}$ and which slope gives $\overline{\mathrm{E}}$.

\section{SPRAYED PARTICLES FLATTENING AND SPLAT COOLING}

\subsection{Measuring techniques}

As emphasized in the introduction, plasma sprayed coating properties depend strongly upon the contact between the layered lamellae[7,8]. Study this layering is not a simple problem due to the particle trajectory distribution[4] and their small size. For example when spraying alumina particles $30 \mu \mathrm{m}$ in diameter at a rate of $3 \mathrm{~kg} / \mathrm{h}$ their injection rate is $2.10^{7} \mathrm{part} / \mathrm{s}$ ! and they impact on the substrate with velocities between 150 and $300 \mathrm{~m} / \mathrm{s}$. For now 5 years many efforts have been devoted to the development of fast pyrometers $(\sim 50-100 \mathrm{~ns})$ to follow temperature and surface evolution during particle flattening and the resulting splat cooling together with flattening and cooling models[5,34].

a) First techniques were developed to collect the particles within the spray cone without overlapping and without[35] or with[36] substrate temperature control.

By optical or scanning electron microscopy coupled with image analysis the splats diameter and shape factor distributions can be determined all over the spray cone.

b) The first pyrometer to study particle flattening was developed in 1992[37] at CNRC Boucherville Canada and then improved regularly[38-40]. The last system consists of a Phase Doppler Particle Analyser (PDPA) and a pyrometer as shown in fig. 8.a. impact.

The PDPA system make it possible to measure the velocity and size of a single particle before its

The size and velocity of a particle in-flight are determined from the light scattered by the particle passing through an interference fringe pattern generated by the intersection of two coherent laser beams whose wavelength was $514.5 \mathrm{~nm}$. The velocity is deduced from the frequency of the Doppler burst generated by the particle crossing the fringe pattern (see fig. 8.b). The size is computed from the phase shift between the light signals collected by two sensors looking at the probe volume from two different angles. This phase shift its linearly related to the particle size. In the PDPA system used in this work, the two sensors are avalanche diodes. After amplification, the signals from the diodes are recorded using a digital oscilloscope. Algorithms based on a cross spectral density method are used to obtain the frequency and the phase shift of signals. The probe volume is limited using a spatial filter placed before the photodetectors. It corresponds to a cylinder $(1.5 \mathrm{~mm}$ long and $0.4 \mathrm{~mm}$ in diameter) whose axis is orthogonal to plasma jet axis. Velocities between $50 \mathrm{~m} / \mathrm{s}$ and $500 \mathrm{~m} / \mathrm{s}$ can be measured for particles which diameters are between $5 \mu \mathrm{m}$ and $100 \mu \mathrm{m}$. 


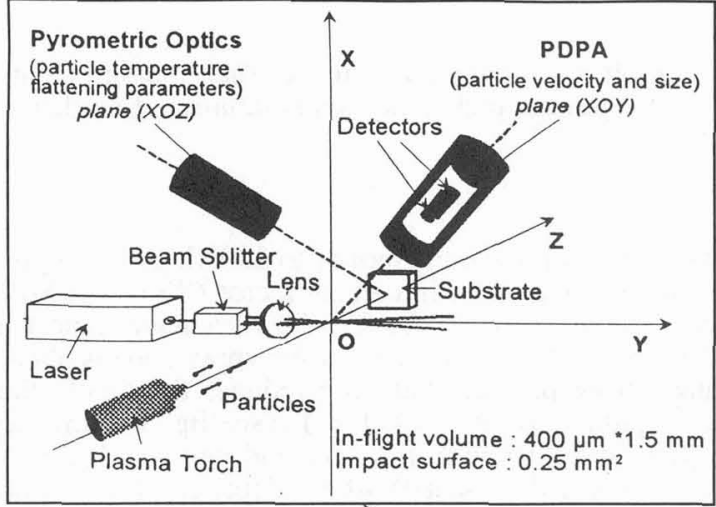

a)

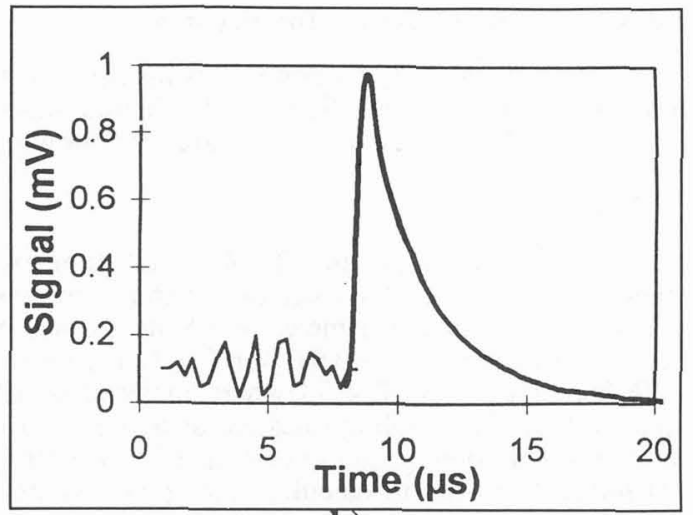

b)

Figure 8 : a) Schematic view of the experimental set-up for the monitoring of particles impinging on a surface[40], b) typical signals.

The temperature of the particle before its impact, the time-temperature evolution of the resulting splat and the flattening parameters (flattening time and flattening degree) are determined from the thermal radiation emitted by the particle in flight and at impact [40] (see fig. 7.b). This radiation is collected by a pyrometer head focused onto the substrate. The monitored area on the substrate surface was about $0.5 \mathrm{~mm}^{2}$. The temperature range of the set up is between $2000 \mathrm{~K}$ and $4000 \mathrm{~K}$.

The flattening time is deduced from the rise time of the signals upon impact. The temperature of a particle just before its impact and that of the resulting splat are derived, after calibration, from the ratio of the photodetector outputs of the pyrometer, by assuming that the material behaved as a grey body. As the signal is proportional to the cross section of the emitted area, the flattening degree is estimated from the square root of the ratio signals just before and upon impact after a correction for temperature effect. When significant cooling takes place during the flattening processes, the collected signal is diminished. However, in that case it is possible to determine the flattening degree for the resulting collected diskshaped splats by measuring their thickness and diameter.

The mean cooling rate of the splat after the spreading process is complete, is estimated from the time-temperature evolution of the lamella.

c) The collected splats, coming from impacting particles whose history of temperature, velocity and diameter is known, are examined by Atomic Force Microscopy (AFM) which allows to determine the size of the columnar structure (see fig. 9.a), the area of poor contact (see fig. 9.b), the columns coalescence[41].

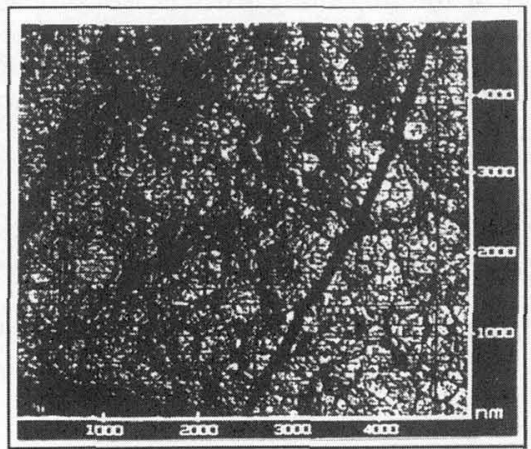

a)

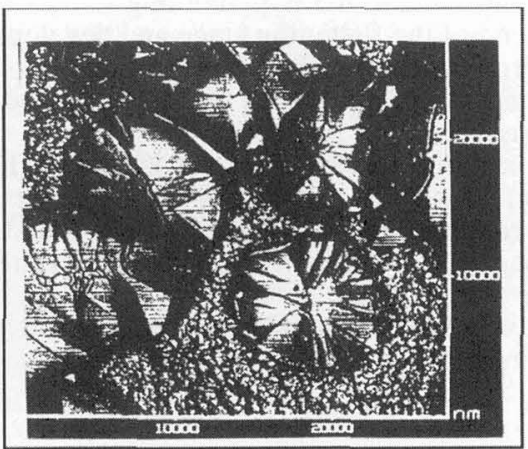

b)

Figure 9 : AFM view of an alumina splat sprayed with a d.c. torch (7 mm) : a) on previously deposited alumina, b) on stainless steel $304 \mathrm{~L}$ (the "big crystals" correspond to a poor contact area)[41]. 


\subsection{Characteristic results for zirconia}

For given spraying conditions resulting in fully molten particles upon impact such measurements have shown the drastic influence of substrate material, roughness, preheating temperature and oxidation stage $[5,36,39,42,43]$. The main results are the following :

\section{a) Single splat}

A critical temperature $T_{c}$ of the substrate exists. For zirconia it corresponds to $200^{\circ} \mathrm{C}$. Below $T_{c}$ on smooth $(\mathrm{Ra}<0.1 \mu \mathrm{m})$ substrates the splats are extensively fingered with a shape factor $(\mathrm{SF}=4 . \Pi$.S/P2 where $P$ is the splat perimeter and $S$ its surface area) below 0.6 while for $T>T_{c}$ they have almost a perfect disk shape $(\mathrm{SF}>0.9)$. For $\mathrm{T}>\mathrm{T}_{\mathrm{c}}$ the mean diameter of the splats all over the spray cone is about $40 \%$ higher than for $\mathrm{T}<\mathrm{T}_{\mathrm{c}}$ where material splashing during particle flattening reduces drastically the splat surface. The cooling rates are also one order of magnitude lower when $T<T_{c}$ (see fig. 10 showing the measured cooling rate evolution with impacting particle temperature fig. $10 . \mathrm{a}$ and velocity $10 . \mathrm{b}$ ). A 1D model[5] allows to calculate the splat cooling (assuming a disk shape) which depends on the splat

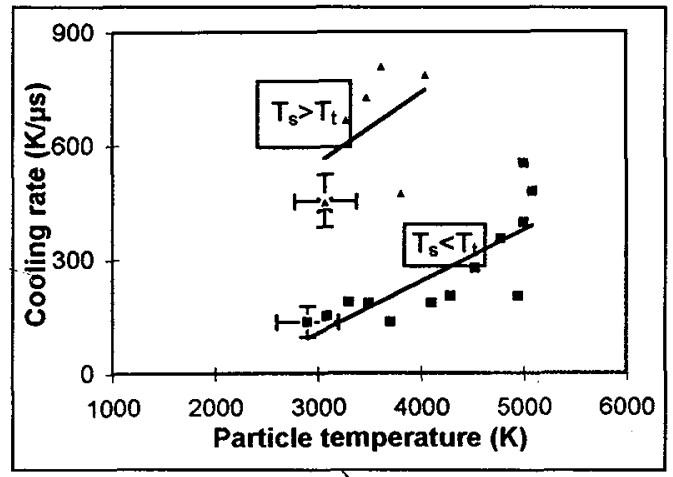

a)

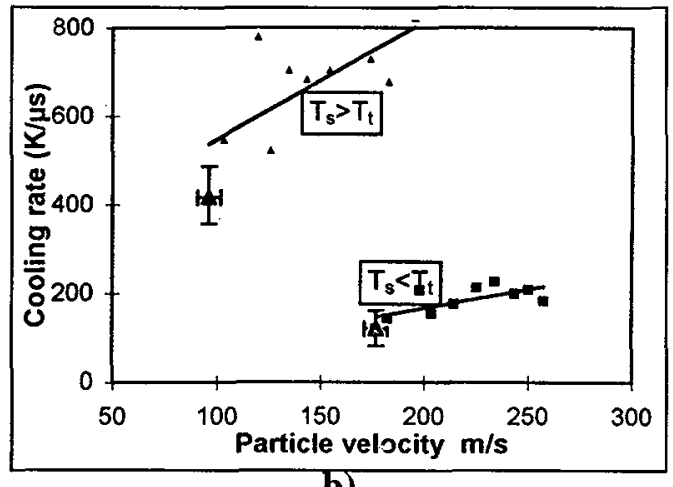

b)

Figure 10 : Evolution of the splat cooling rate with zirconia impacting particle : a) temperature and b) velocity for two substrate preheating temperatures.

thickness (decreasing with increasing impact velocity) and on thermal contact resistance $\mathbf{R}_{\text {th }}$ between the splat and substrate or previously deposited layers. By comparing the measured and calculated cooling rates for $\mathrm{T}>\mathrm{T}_{\mathrm{c}}$, knowing the splat thickness, it allows to determine $\boldsymbol{R}_{\mathrm{th}}$ which in this case is of the order of 10-7 $-10^{-8} \mathrm{~m}^{2} . \mathrm{K} / \mathrm{W}$ (almost a perfect contact). The explanation for $\mathrm{T}_{\mathrm{c}}$ has been given by $\mathrm{A}$. Vardelle et al[44]. If the substrate surface temperature $T_{i}$ (resulting from preheating and flattening particle heat transfer) is higher than the hypercooling temperature $T_{H}$ of the impacting particle, solidification occurs only at the end of the flattening stage and develops all over the splat surface resulting in an homogeneous columnar growth. If $\mathrm{T}_{\mathrm{i}}<\mathrm{T}_{\mathbf{H}}$ solidification starts at a few area before flattening is completed and the flowing liquid material, blocked by this solidified area, splashes away. Preheating at $T_{c}$ allows to obtain or not the case $T_{i}>T_{H}$.

The cooling rate of the splat is closely linked to $T_{c}$ and splat thickness. The size of the splat columns increases when the cooling rate decreases.

On rough surfaces, as the flowing liquid material of the impacting particle is blocked by the surface asperities it results in thicker splats. Here again the influence of $T_{c}$ is drastic the cooling rates being higher for $\mathrm{T}>\mathrm{T}_{\mathrm{c}}$.

The way substrate oxidation modifies the splat shape even for $T>T_{c}$ is not yet clearly understood but works are in progress[45].

\section{b) Layered splats}

In classical spraying conditions where a pass corresponds to 3 to $10 \mu \mathrm{m}$ (about 3 to 10 layered splats) and where temperature between successive passes is in the range of $500-600^{\circ} \mathrm{C}$ the results obtained for $T_{c}$ have been confirmed. With a preheating temperature over $T_{c}$ the adhesion/cohesion of the coatings is more than doubled[4],46]. When increasing the preheating temperature over $T_{\mathfrak{c}}$ and the pass thickness, temperatures in the range of $900-1000^{\circ} \mathrm{C}$ within the coating are reached and the new impacting particle starts to nucleate only at the end of the flattening stage. Thus the columnar structure of the previously 
deposited and solidified splat acts a a nucleation site and it results in a columnar structure growing through the whole pass and between passes[47]. However a compromise has to be found with the increase of the stresses[40] and work is in progress in this field.

\section{CONCLUSION}

Arc root restrike mode in d.c. plasma torches has a great influence on their working conditions especially for plasma spraying. It results in :

- important power fluctuations (up to $50 \%$ ), due to voltage variations, at frequencies between a few $\mathrm{kHz}$ and $20 \mathrm{kHz}$. This frequency depends strongly on the hot boundary layer formation around the arc column. If its correlation with the torch working parameters is now well understood for axial gas injection, the effect of gas vortex injection requires more studies.

- a discontinuous behavior of the plasma which can be described by a succession of hot puffs travelling with the flow and surrounded by colder layers.

- the possibility to use these transient phenomena to measure the plasma flow velocity by analyzing the light signals recorded at two points which distance is known. This requires an appropriate signal treatment, the useful information being kept in the low frequency band pass.

- to determine the mean electric field of the column by comparing the light and voltage signals.

- the necessity to extract the stationary component of the volumetric emission coefficient in order to determine spectroscopically the plasma jet temperature, the classical measurements based on time averaging being incorrect.

However a lot of work has still to be done to :

- extract information from the high frequency (a few $\mathrm{kHz}$ ) voltage signal[33].

- determine the contribution of this piston flow (succession of puffs) on the plasma flow modelling including the engulfment process of the surrounding air entrainment $[48]$.

If the transient phenomena in d.c. plasma torches start to be well understood their consequences on the injected particles treatment has not yet been studied and work is in progress to :

- evaluate the influence of these puffs and their frequency on the sprayed particles heating by the plasma jet, the first measurements[49] showing that the flux emitted by the hot particles fluctuates too.

- find a way to determine statistically the particles treatment within the jet and how it can affect the splats formation and their layering.

If in the eighties many studies were focused on the measurement of particle surface temperature, velocity and diameter distribution, during their flight within the plasma jet, it is only for about 5 years that the study of their flattening and the resulting splat cooling has been developed. Fast pyrometers $(<100 \mathrm{~ns})$ associated with laser scattering measurements have allowed to determine for a single particle its temperature, velocity and diameter prior to its impact, its flattening degree and time $(\sim 1 \mu \mathrm{s})$ as well as the resulting splat temperature evolution. Such measurements have allowed to understand better the drastic influence of the substrate temperature, roughness and oxidation stage on splats adhesion and work is in progress to study their layering in order to achieve coatings with better thermomechanical properties.

\section{References}

[1] P. Fauchais and M. Vardelle, Pure and Applied Chemistry, 66 (1994) 1247-1258.

[2] Thermal Spraying (Pub.) American Welding Soc. Miami, FL, USA (1985), 180 p.

[3] L. Pawlowski, The science and engineering of thermal spray coatings (Pub.) J. Wiley and sons, NY, USA (1995), $286 \mathrm{p}$.

[4] M. Vardelle, A. Vardelle and P. Fauchais, J. of Thermal Spray Technology, 2 (1) (1993) 79-92.

[5] M. Vardelle, A. Vardelle, A.C. Leger, P. Fauchais and D. Gobin, J. Thermal Spray Technology, 4 (1) (1995) $50-58$.

[6] S.A. Wutzke, E. Pfender and E.R.G. Eckert, AIAA Journal, $\underline{5}$ (4) (1967).

[7] R. McPherson; Thin Solid Films, 83 (1981) 297-310.

[8] R. McPherson, Surf. Coating Technol., 39/40 (1989) 173-181.

[9] C. Moreau, P. Cielo, M. Lamontagne, S. Dallaire and M. Vardelle, Meas. Sci. Technology, 1 (1990) 807-817.

[10]W. Neumann, Beitr. Plasma Physik, 16 (1976) 97-109.

[11] M.F. Zhukov et al, Electric arc gas heaters (plasma torches) (Pub.) Nauka, Moscow (1973) 232 p (in Russian).

[12] M.F. Zhukov, in Thermal Plasma and New Materials Technology, (eds.) O.P. Solonenko and M.F. Zhukov, (Pub.) Cambridge Interscience (1994) 9-43.

[13] J.F. Brilhac, B. Pateyron, G. Delluc, J.F. Coudert and P. Fauchais, Plasma Chemistry, Plasma Proc., 15 (1) (1995) 231-255. 
[14] M.P. Planche, Z. Duan, O. Lagnoux, J. Heberlein, J.F. Coudert, E. Pfender, Study of arc fluctuations with different plasma spray torch configurations, to be published in proc. of ISPC 13, (ed.) Prof. W.K. Wu, Beijing, Aug. (1997).

[15] J.F. Coudert, M.P. Planche and P. Fauchais, High Temp. Chem. Processes 3 (1994) 639-651.

[16] J.F. Coudert, M.P. Planche and P. Fauchais, in Heat and Mass Transfer under Plasma Conditions (ed.) P. Fauchais, (Pub.) Begell House, N.Y., USA (1994) 253-264.

[17] J.F. Coudert, M.P. Planche and P. Fauchais, Time resolved measurements of arc voltage, temperature and velocity in a d.c. plasma torch, in Proc. ISPC12 (ed.) Prof. J. Heberlein, Univ. of Minnesota MN, USA $\underline{3}$ (1995) 1619-1626.

[18] M.P. Planche, Contribution à l'étude des fluctuations dans une torche à plasma, Application à la dynamique de l'arc et aux mesures de vitesse de l'écoulement, Ph.D. Thesis, Univ. of Limoges, France (1995).

[19]E. Pfender, Electric Arcs and Arc Gas Heaters, in Gaseous Electronics (eds.) M.N. Hirsch and H.J. Oskam (Pub.) Academic Press 1 (1978) 291-345.

[20] O.I. Yas'ko, Brit. J. Appl. Phys. 2 (2) (1969) 733-741.

[21]M. Boulos, P. Fauchais and E. Pfender, Thermal Plasma, Vol. 1 Fundamental phenomena, (Pub.) Plenum Press (1995) $452 \mathrm{p}$.

[22] J.M. Sobrino, J.F. Coudert and P. Fauchais, Anodic arc root behavior of a transferred arc moving orthogonally to a plume surface, in proc. of ISPC 12 (ed.) J. Heberlein, Univ. of Minnesota $\underline{3}$ (1995) 1455-1460.

[23]X. Zhou and J. Heberlein, Plasma Sources Sci. Technol. 3 (1994) 564-574.

[24] X. Zhou and J. Heberlein, in Proc. of Symp. on heat and mass transfer under plasma conditions (ed.) P. Fauchais, (Pub.) Begell House Inc., NY, USA (1995) 237-243.

[25] X. Zhou and J. Heberlein, Plasma Chem. Plasma Proc. (1996) 229S-244S.

[26] X. Zhou, B. Ding and J. Heberlein, IEEE Trans. Components Pkg. and Mfg. Tech. 19 (3) (1996) 320328.

[27] M. Ushio, K. Tanaka and M. Tanaka, see [23] (1995) 265-272.

[28] J.F. Coudert, M.P. Planche and P. Fauchais, Plasma Chem. Plasma Proc. 15 (1) (1995) 47-70.

[29] M.P. Planche, J.F. Coudert and P. Fauchais, see [21] $\underline{3}$ (1995) 1475-1480.

[30] B. Pateyron, M.F. Elchinger, G. Delluc and P. Fauchais, Plasma Chemistry Plasma Processing 16 (1) (1996) 39-57.

[31]E. Pfender, J. Fincke and R. Spores, Plasma Chem. Plasma Process, 11 (4) (1991) 529-544.

[32] J.F. Coudert and P. Fauchais, J. of High Temp. Chem. Processes 3 (1) (1992) 443S-453S.

[33] J.F. Coudert, M.P. Planche and P. Fauchais, Plasma Chem. Plasma Process. 16 (1) (1996) 211S$228 \mathrm{~S}$.

[34]P. Fauchais, A.C. Leger, M. Vardelle and A. Vardelle, Formation of plasma sprayed oxide coatings, in Proc. of J. Szekely Memorial Symposium (Oct. 1997) to be published by TSM.

[35] K.A. Roberts and T.W. Clyne, Surface and Coating Technology 41 (1990) 103-115.

[36] L. Bianchi, A. Grimaud, F. Blein, P. Lucchese and P. Fauchais, J. Thermal Spray Technology 4 (1) (1995) 59-66.

[37] C. Moreau, P. Cielo and M. Lamontagne, J. of Thermal Spray Technology 1 (3) (1992) 317-323.

[38] M. Vardelle, A. Vardelle, C. Moreau and P. Fauchais, Meas. Sci. Technol. $\underline{5}$ (1994) 205-212.

[39] S. Fantassi, M. Vardelle, P. Fauchais and C. Moreau, J. Thermal Spray Technol. 2 (4) (1993) 379384.

[40]A.C. Leger, Contribution to experimental study of zirconia splat formation, splats layering and coating residual stresses, Ph.D. Thesis, Univ. of Limoges, March (1997).

[41] L. Bianchi, A. Denoirjean, F. Blein and P. Fauchais, accepted in Thin Solid Films (1997).

[42] L. Bianchi, A.C. Leger, M. Vardelle, A. Vardelle and P. Fauchais, accepted in Thin Solid Films.

[43] C. Moreau, P. Gougeon and M. Lamontagne, J. Thermal Spray Technol. 4 (1) (1995) 25-33.

[44] A. Vardelle, N.J. Themlis, B. Dussoubs, M. Vardelle and P. Fauchais, Transport and Chemical rate phenomena in plasma sprays, accepted in J. of High Temp. Material Proc. $\underline{3}$ (1997).

[45] A. Denoirjean, L. Bianchi and P. Fauchais, Study of oxide layers obtained on 304L substrates heated by a d.c. plasma jet plume, to be published in Proc. of UTSC 97 (Pub.) ASM Int. OH USA.

[46] P. Fauchais, M. Vardelle, A. Vardelle, L. Bianchi and A.C. Leger, Plasma Chem. Plasma Process. 16 (1) (1996) $99 \mathrm{~S}-126 \mathrm{~S}$.

[47] A. Haddadi, A.C. Leger, A. Grimaud, P. Fauchais and F. Nardou, Influence of substrate and coating temperature on columnar growth in Proc. of UTSC 97 (Pub.) ASM Int. OH, USA.

[48]E. Pfender, J. of Thermal Spray Technology 6 (2) (1997) 126-128.

[49] K.I. Li, M. Vardelle and P. Fauchais, in Advances in Thermal Spray Science and Technology (eds.) C.C. Berndt and S. Sampath (Pub.) ASM Int. OH, USA (1995) 45-50. 